\title{
Tunable bands in biased multilayer epitaxial graphene
}

\author{
Michael D. Williams, ${ }^{a}$ Duminda K. Samarakoon, ${ }^{b}$ Dennis W. Hess ${ }^{c}$ and Xiao-Qian Wang $* a$ \\ Received 14th December 2011, Accepted 28th February 2012 \\ DOI: 10.1039/c2nr11991a
}

\begin{abstract}
We have studied the electronic characteristics of multilayer epitaxial graphene under a perpendicularly applied electric bias. Ultraviolet photoemission spectroscopy measurements reveal that there is notable variation of the electronic density-of-states in valence bands near the Fermi level. Evolution of the electronic structure of graphite and rotational-stacked multilayer epitaxial graphene as a function of the applied electric bias is investigated using first-principles density-functional theory including interlayer van der Waals interactions. The experimental and theoretical results demonstrate that the tailoring of electronic band structure correlates with the interlayer coupling tuned by the applied bias. The implications of controllable electronic structure of rotationally fault-stacked epitaxial graphene grown on the $\mathrm{C}$-face of $\mathrm{SiC}$ for future device applications are discussed.
\end{abstract}

\section{Introduction}

Graphene, a monolayer of $\mathrm{sp}^{2}$-hybridized carbon, is the subject of much interest in the fields of optoelectronics, sensors, and hydrogen storage. ${ }^{1-8}$ For instance, the high carrier mobility suggests that this material may be a viable successor for copper interconnects in electronic device structures. The electronic properties have been shown to be tunable from metallic to semiconducting with hydrogen intercalation. ${ }^{3}$ However, many potential applications for graphene require ordered growth on an insulating substrate. One successful methodology to produce graphene layers has been thermal decomposition of $\mathrm{SiC}$ in a vacuum. ${ }^{4}$ Large graphene grain sizes have also been reported via thermal decomposition of $\mathrm{SiC}$ in an inert gas atmosphere. ${ }^{5}$ Other studies have explored the processing and growth of epitaxial graphene (EG) layers using biological and chemical functionalization methodologies. ${ }^{6}$ The latter approach is particularly attractive for sensor and hydrogen storage applications. ${ }^{7}$

In virtually all potential applications of graphene, an understanding of the electronic properties of graphene is critical to successful integration of graphene into future nanoelectronic devices. ${ }^{8-17}$ In this regard, interlayer interactions in multilayer epitaxial graphene (MEG) play an important role in determining the electronic structure characteristics. ${ }^{18-34}$ The planar nature of MEG allows analysis by traditional surface science techniques. ${ }^{16}$ In this work, we use angle integrated ultraviolet photoemission spectroscopy (UPS) to monitor the effect of sample bias on the joint density-of-states (DOS) of MEG and highly ordered

${ }^{a}$ Department of Physics and Center for Functional Nanoscale Materials, Clark Atlanta University, Atlanta, Georgia 30314, USA. E-mail: xwang@cau.edu

${ }^{b}$ Department of Chemistry, Clark Atlanta University, Atlanta, Georgia 30314, USA

${ }^{c}$ School of Chemical and Biomolecular Engineering, Georgia Institute of Technology, 311 Forest Drive, N.W., Atlanta, GA 30332-0100, USA pyrolytic graphite (HOPG). The MEG is grown on the C-face of $\mathrm{SiC}$ and possesses properties of an isolated graphene sheet owing to the peculiar stacking-order of the multilayers. ${ }^{13,14}$ UPS is an ideal tool for studying interlayer interactions due to its ability to extract the joint DOS for valence bands in the first two layers of samples. Furthermore, we employ a dispersion-corrected density-functional theory (DFT) ${ }^{33}$ that incorporates interlayer van der Waals interactions to investigate the corresponding electronic structure. ${ }^{34}$ The calculated dependence of the electronic structure on the applied electric bias is in good conformity with experimental observations in that there is a notable change of the electronic DOS in the proximity of the Fermi level, and the DOS for MEG displays a more prominent increase relative to that for HOPG.

\section{Results and discussion}

In graphite, the characteristic linearly dispersing bands of monolayer graphene are superseded by pairs of split hyperbolic bands associated with Bernal stacking. The Bernal-stacked layers are electronically coupled in a manner similar to that in graphite, which lacks the appealing electronic properties of individual graphene sheets. ${ }^{29-34}$ The parabolic bands correspond to charge transport by massive fermions, whereas the electrons in single layer graphene behave as massless Dirac fermions. ${ }^{13,14,30,31}$ Experimental studies to date for MEG on $\mathrm{C}$-face $\mathrm{SiC}$ indicate that the electronic states of the layers are decoupled as a consequence of rotational fault stacking. ${ }^{13,14}$ A variety of experimental and theoretical studies show that rotational stacking order in MEG leads to decoupling of the layers and a linear dispersion band structure consistent with single-layer graphene. ${ }^{13,14,21}$ Therefore, MEG grown by thermal decomposition on $\mathrm{SiC}$ substrates and patterned via standard lithographic procedures has been proposed as a platform for carbon-based nanoelectronics and molecular electronics. ${ }^{13,14}$ Consequently, the 
characteristics of the electrons in MEG are expected to vary sensitively with interlayer coupling. An in-depth understanding of the interlayer coupling is thus of crucial importance to tailor the electronic properties of MEG.

To pursue the effect of interlayer coupling experimentally, we collected UPS spectra of MEG and HOPG samples under an applied electric field. Fig. 1 shows the corresponding UPS spectra, which are normalized to the low kinetic-energy peak near the photoemission threshold associated with the low energy scattered electrons. The spectra have been shifted in energy such that the photoemission thresholds coincide with that of the $-1.0 \mathrm{~V}$ biased spectrum. The thresholds of photoemission are determined by linearly extrapolating the low kinetic energy edge of each electron distribution curve (EDC) from the full width at half maximum of the low energy spectral peak to the spectral baseline. It is worth noting that these shifts are non-electrostatic in nature; i.e., the shifts are non-rigid. The binding energy scale of the spectra is referenced to the Fermi level of the UPS system. The Fermi level is the maximum energy of photoemitted electrons in the EDC of elemental indium in the system. The nonrigid nature of the shifts makes it necessary to align the EDC thresholds, and indicates the existence of band structure modifications of MEG and HOPG with applied bias.

The $\mathrm{sp}^{2}$-feature centered at $-10.5 \mathrm{eV}$ for the grounded graphene sample is associated with the crystalline state of the material. ${ }^{9}$ The $\mathrm{sp}^{2}$ band structure related to the valence band maximum (VBM) is affected by the morphology of the surface, since the structural defects disrupt the $\mathrm{sp}^{2}$ network. We show here that the band structure is also affected by external bias. EDC emissions for the sample peak in the valence band of graphene increase with amplified negative sample bias such that the centroid of the peak is $9.85 \mathrm{eV}$ below the Fermi level.

In the absence of an electric bias, the measured EDCs for HOPG and MEG are similar in that there is a monotonic increase of the electronic DOS starting at the Fermi level, while the predominant increase emerges from $-8 \mathrm{eV}$. With the application of a perpendicular electric bias, the corresponding UPS signals undergo reversible changes that are distinctive from simple shifts. Specifically, for HOPG, there is a slight change of the DOS in the vicinity of the Fermi level $(0 \mathrm{eV})$. The predominant changes are manifested in the region of $2-9 \mathrm{eV}$ below the
Fermi level in that the DOS raises in this region with the increase of applied electric field. In contrast, for MEG, there is a marked increase of the DOS starting at the Fermi energy. Furthermore, the shifts of the central peak for MEG are more pronounced than in the HOPG counterpart. It appears that for HOPG, an electric field of $3 \mathrm{~V}$ is the transition point that separates strong and weak field effects. As can be seen from Fig. 1a, for the three measured EDCs with 0-2 V electric bias, the curves show analogous trends, while the other spectra corresponding to $3-10 \mathrm{~V}$ applied bias collectively show different behavior. For comparison, the EDC for MEG (Fig. 1b) shows systematic evolution with the applied electric bias.

It is worth noting that in the proximity of the Fermi level the effect of electric field on the DOS is distinctive for HOPG and MEG. Specifically, for HOPG, the changes are insignificant in the range of $0-1 \mathrm{eV}$ below the Fermi level (between the two dashed lines in Fig. 1). Furthermore, the density of charge carriers can be tuned in a similar manner. In contrast, for MEG, the slope of the electronic DOS changes proportionally with the applied electric field. Such differences in the electronic DOS between HOPG and MEG are closely connected with the electronic structure characteristics. An important ramification of the UPS investigation is that the applied electric field greatly affects the interlayer coupling, and the distinctive electronic structure characteristics between HOPG and MEG can be attributed to their peculiar stacking orders. This suggests that the transport behavior of devices can be modified with the simple application of an electric field.

Recent experiments have indicated that Bernal-stacked bilayers constitute a small fraction of the adjacent layers in multilayer stacks. ${ }^{13,14}$ Angle-resolved photoemission experiments have provided unambiguous evidence that the band structure for twisted adjacent layers remains identical to that of isolated graphene. ${ }^{13,14}$ The role of stacking symmetry in determining the band structure of graphene sheets can be established by comparing graphene with distinctive stacking arrangements. In graphite, the Bernal-stacked graphene sheets are rotated $\pi / 6$ relative to adjacent sheets in the stack. Bernal stacking results in the fact that the two atoms in a graphene unit-cell (labeled as ' $\mathrm{A}$ ' and ' $\mathrm{B}$ ', respectively) are not equivalent. The stacking-induced disparity of the ' $\mathrm{A}$ ' and ' $\mathrm{B}$ ' atoms in graphite is generally referred
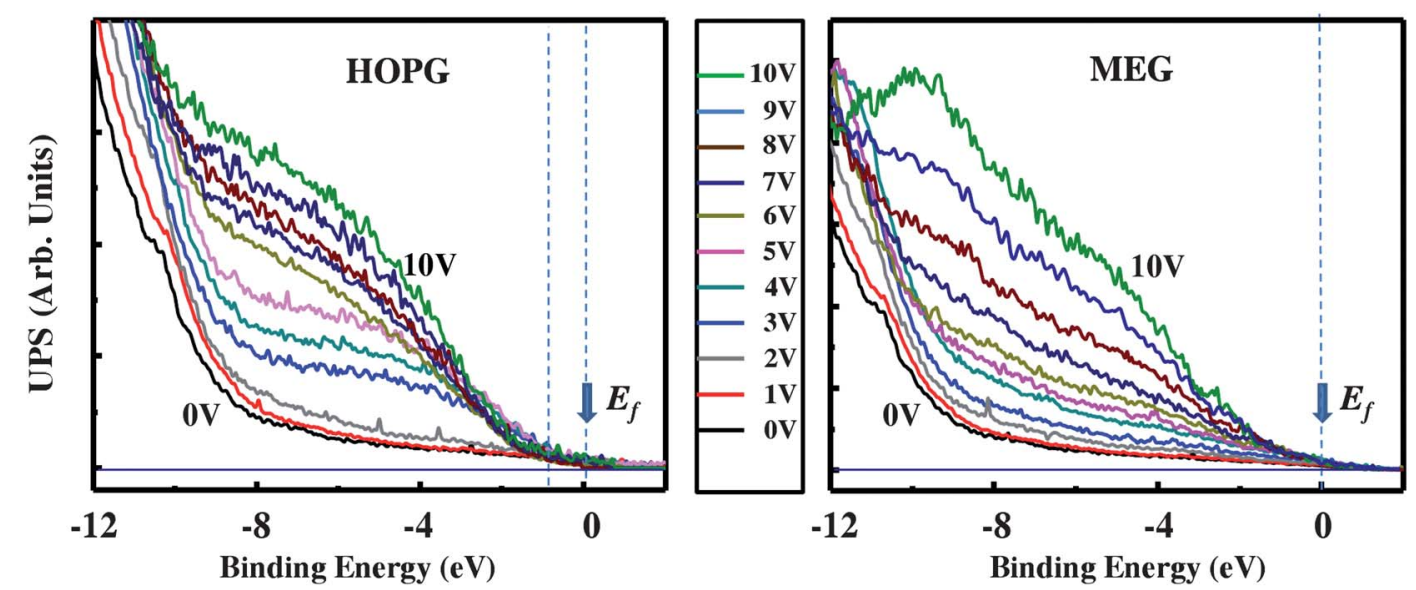

Fig. $121.2 \mathrm{eV}$ UPS of HOPG (left panel) and MEG (52 A thick) on SiC (0001) (right panel) with 0-10 V electric bias. 
to as $\mathrm{AB}$ stacking. The energy bands of rotationally faulted multilayers disperse nearly linearly with momentum in the proximity of the Dirac point. As such, the electronic structure of MEG is largely determined by the rotationally faulted characteristic.

To facilitate an understanding of the experimentally observed electronic DOS features, we have employed a dispersion-corrected DFT approach. To model the MEG, we used a commensurate twisted bilayer with a rotational angle of $21.8^{\circ} .{ }^{30,31}$ The atomistic schematics of Bernal stacking graphite and twisted bilayer graphene are depicted in Fig. 2a and b, respectively. These configurations have parabolic and linear dispersion relations, and thus are viewed as prototypes for representing HOPG and MEG, respectively. Graphite and twisted bilayer with $21.8^{\circ}$ rotation are both odd in sublattice exchange. ${ }^{31}$ As a result, the characteristic band structure is semi-metallic in that the conduction and valence bands touch at the Fermi level. However, in contrast to the parabolic dispersion for graphite, the twisted bilayer shows a linear dispersion that is attributed to weak interlayer coupling. ${ }^{30,31}$ Upon the application of an electric bias, the electronic states near the Fermi level for graphite (Bernal stacking) are little affected. This is attributed to the constraints associated with the sublattice exchange, and the fact that the electric bias perpendicular to the graphite layers preserves the symmetry. ${ }^{31}$ However, the situation is different for the twisted layers in that there are $\mathrm{AA}$ and $\mathrm{AB}$ stacking regions. ${ }^{30,31}$

The rotationally faulted stacking in C-face MEG leads to manifestly distinct electronic structure from that of the Si-face epitaxial graphene (EG). ${ }^{13,14}$ Si-face EG is Bernal stacked analogous to graphite, which is grown with both a buffer-layer and a top graphene layer. Only the top layer is isolated and exhibits the band structure of a single graphene sheet. ${ }^{13,14}$ When $\mathrm{H}_{2}$ is intercalated into the interface, the buffer layer becomes isolated from the substrate, and the two graphene layers develop into a novel electronic system. Because the buffer layer is rotated $\pi / 6$ relative to the top layer with Bernal stacking, the doped Dirac cone of the single layer converts into split bilayer bands in the structure of a bilayer pair. However, the buffer layer plays an
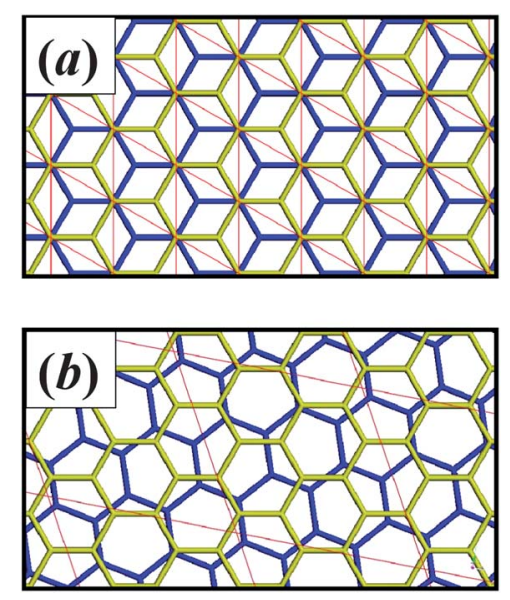

Fig. 2 Top views of (a) AB stacked graphite and (b) twisted adjacent layers of graphene with a $21.8^{\circ}$ twist angle. First and second layers are colored with blue and gold, respectively. important role in the electronic structure as there is nontrivial symmetry change to the Bernal stacking bilayer system. On the other hand, the majority stacking in C-face MEG is nongraphitic so that films with as many as 60 graphene layers still behave electronically in a manner similar to that of a stack of isolated graphene sheets. ${ }^{13,14}$

To assess the effect of stacking order on the interlayer coupling, we can look at the band structure of bilayer graphene when interlayer interactions are present while the $\mathrm{AB}$ stacking symmetry is absent. The simplest instance is AA stacking in which two graphene layers stack directly on top of each other. In the AA stacking, the sublattice exchange is of even symmetry and the interlayer electronic coupling is suppressed by a significant Pauli repulsion arising between the graphene layers. Consequently, the dispersion in AA stacking leads to multiple linear bands of graphene. ${ }^{31}$ This effect is general and applies to rotationally faulted bilayers. ${ }^{31,32}$ The change in sublattice exchange symmetry leads to dramatic modifications in the electronic band structure of rotationally faulted graphene sheets. For our purpose, we employed a model of two graphene sheets rotated by $21.8^{\circ}$ to investigate the electronic structure of MEG. The $21.8^{\circ}$ rotationally faulted bilayer has the smallest commensuration unit cell.

We show in Fig. 3 the calculated band structures for graphite and twisted bilayer, respectively. As seen in Fig. 3a, the graphite has two parabolic bands at the Dirac point $(\mathrm{K})$ separated by $1.08 \mathrm{eV}$ and $1.32 \mathrm{eV}$ for conduction and valence bands, respectively. With the application of electric bias, the split conduction and valence bands merge (comparison highlighted by green and brown circles in Fig. 3a-c). At an electric field of $\varepsilon=0.51 \mathrm{~V}^{-1}$, the two valence bands become almost degenerate. For MEG, on the other hand, the linear dispersion bands at the Dirac point are manifest in the absence of the electric bias. Upon applying the electric bias, the linear-dispersed bands merge and hybridize, thereby changing the band topology and resulting in a metallic state. As can be seen from Fig. 3d-f, the radius of the Fermi
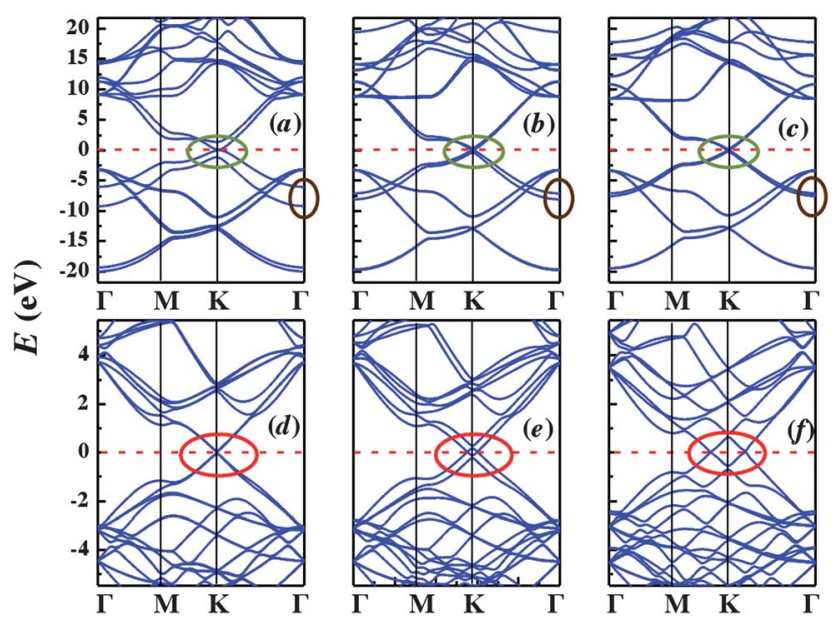

Fig. 3 Calculated band structure (a-c) for graphite and (d-f) for twisted bilayer graphene of commensurate fault at $21.8^{\circ}$ with (from left to right) $0,0.26$, and $0.51 \mathrm{~V} \AA^{-1}$ electric field, respectively. $\Gamma=(0,0), K=(-\pi / 3 a$, $2 \pi / 3 a)$, and $M=(0, \pi / 2 a)$, where $a=6.46 \AA$ for twisted bilayer graphene and $2.48 \AA$ for graphite. The Fermi level, highlighted by a dashed red line, is shifted to $0 \mathrm{eV}$. 
surface increases with increasing electric bias, yielding a distinctly non-zero electronic DOS. Specifically, the single Dirac cone splits into two Dirac cones and the separation of the two cones in the momentum space is proportional to the applied electric bias (highlighted by red circles in Fig. 3d-f). An important consequence is that the electronic DOS near the Fermi level increases proportionally with the electric bias. Interestingly, the extracted band structure at finite electric bias closely resembles the band structure of AA stacking, particularly regarding the appearance of doubled Dirac cones. ${ }^{31}$

In the vicinity of the Fermi level, the theoretically predicted changes in electronic DOS for graphite and the twisted bilayer of commensurate fault at $21.8^{\circ}$ are in good agreement with experimentally measured UPS for HOPG and MEG, respectively. Specifically, the effect of electric bias on the states near the VBM is manifested in the merge of split valence bands for graphite and the creation of doubled Dirac cones for the twisted bilayer, respectively. The fuse of split valence bands in graphite is within the low DOS range of $1.32 \mathrm{eV}$ (see Fig. 3a), which corresponds to minor changes in the measured UPS signals for HOPG. The split and doubling of the Dirac cone for the twisted bilayer, however, imply a proportional increase of the electronic DOS with the applied electric bias. This trend agrees with the experimental observations of an increased slope in the UPS of MEG.

We illustrate in Fig. 4a and b the extracted electronic DOS for graphite and the twisted bilayer, respectively. As seen from Fig. 4a for graphite, the electronic DOS increases with applied electric bias in the region from -2 to $-8 \mathrm{eV}$, which is attributed to the merge of split valence bands as indicated in the band structure (Fig. 3a-c). For the twisted bilayer, the effect of zone folding and fuse of split bands is also present, as the calculated electronic DOS increases in the region from -2 to $-8 \mathrm{eV}$ as well (Fig. 4b). Therefore, we attribute the experimentally observed increase of electronic DOS with the applied electric field to the electric-bias-induced band fusing and the associated band flattening behavior.
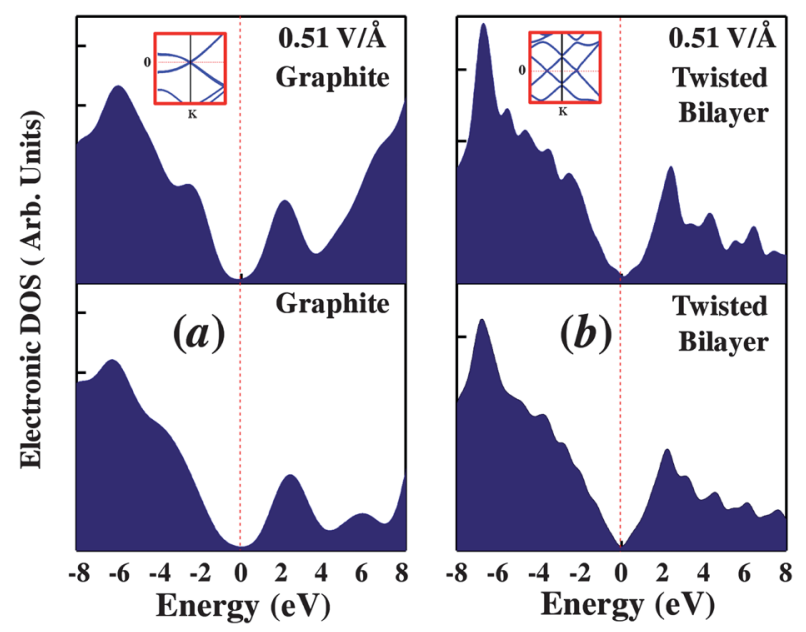

Fig. 4 Calculated electronic DOS for (a) graphite and (b) twisted bilayer graphene with $0.51 \mathrm{~V}^{-1}$ electric bias (top panels) and without electric bias (bottom panels), respectively. Inset: close-up view of the corresponding band structure near the Dirac point.
From the point of view of comparing experiment with theory, the correspondence between the electric field and the bias is interesting in view of the fact that the experimental settings depend on factors that are difficult to estimate. Nevertheless, it appears that an electric field of $0.51 \mathrm{~V}^{-1}$ in the theoretical calculation is in accordance with an experimentally applied bias of $3 \mathrm{~V}$. This estimate is based on the fact that at a field of $0.51 \mathrm{~V}^{-1}$ the split valence bands of graphite become degenerate. Upon further increase of the electric bias, the electronic structure involves band merging among other bands. The extracted band structure for MEG indicates band folding and hybridizations $-3 \mathrm{eV}$ downward from the Fermi level, which appears to support the above estimate.

A few implications of these results should be noted. (i) The twisted bilayer appears to be a reasonable model for representing the electronic structure characteristics of MEG. The $21.8^{\circ}$ rotationally faulted bilayer is the smallest commensurate conformation, which corresponds to the experimentally observed predominant rotationally faulted stacking. The conformation is of odd sublattice exchange symmetry. ${ }^{31}$ Although there are other commensuration cells with even sublattice exchange with distinct band structures, the linear dispersion for the $21.8^{\circ}$ twisted bilayer offers a simple, yet most relevant model. (ii) The split of one Dirac cone into two is reminiscent of the band structure of AA stacking and implies that electric-bias-induced dipole-dipole interactions are more effective with AA stacked carbon atoms. This observation also indicates significant charge transfer between the conduction and valence states due to dipole-dipole interactions. The promotion of AA stacked band structure after application of an electric bias captures the essential physics. (iii) The doubled Dirac cones yield an increase in the number of charge carriers while preserving the linear dispersion near the Fermi level. This feature is important for both practical device applications and the increase of linearly dispersed charge carriers. Moreover, it suggests the possibility of controlling the rotationally faulted layers with the application of an electric bias during the sample growth process. (iv) It is worth mentioning that the dispersion correction is necessary for an accurate description of the structural and electronic properties. Our currently employed dispersion-corrected DFT approach provides quantitatively accurate results for the layer distance, and the effect of electric bias is incorporated in a modified Hamiltonian for electronic structures. ${ }^{35-37}$

\section{Conclusion}

In summary, the evolution of electronic properties of MEG as a function of the applied electric bias has been studied using combined experimental UPS measurements and theoretical dispersion-corrected density functional calculations. The present work demonstrates that significant control of the low-energy electronic states of graphene can be achieved by tuning interlayer interactions in MEG samples. This situation is analogous to the standard description of the 1D band of single-walled carbon nanotubes in which the electronic structure of the set of different nanotubes can be generated by zone folding of the $2 \mathrm{D}$ electronic structure of graphene. Analogous to carbon nanotubes, the additional control of the electronic properties of MEG as a function of electric bias should extend the range of distinctive 
physical phenomena and applications of this material. We remark, before closing, that the interlayer coupling also plays an important role in a variety of graphene-based systems such as hetero-bilayers, ${ }^{38-40}$ which are considered as a promising substrate for device applications. Our results reveal an interesting interlayer coupling tunable by the electric bias, and thus provide useful insight into future applications of a variety of graphenebased materials.

\section{Methods}

The EG sample used in this study was grown by the thermal graphitization of semi-insulating $4 \mathrm{H} \mathrm{SiC}(000 \overline{1})$ wafers in an inductive furnace. ${ }^{14}$ The HOPG was purchased from SPI Supplies. The MEG sample is optically smooth with a thickness of $52 \AA$ and has large areas of contiguous grains that are nominally $2.0 \mu \mathrm{m}$ in size. It was grown at $1560^{\circ} \mathrm{C}$ for 7 minutes on a chemical mechanical polished $\mathrm{SiC}$ substrate that was hydrogen etched prior to the growth of the film.

The HOPG sample was mechanically clamped to a Mo MBE wafer block with Ta wire, and then loaded into the load lock of the UPS analysis system. The EG sample was mounted with In ( $99.9999 \%$ purity) at $160{ }^{\circ} \mathrm{C}$ onto a Mo wafer block in a nitrogen filled glove box at atmospheric pressure. The In completely surrounded the periphery of the EG samples' edges up to the top surface to minimize charging effects. The wafer block was then placed into a sealed container and removed from the glove box for transport in the same laboratory space to the UPS system load lock. The block was removed from the container, placed $(<1$ minute) into the nitrogen purged load lock, sealed, and then pumped down to $<2 \times 10^{-9}$ Torr. UPS analysis was performed after the magnetically coupled transfer of each sample sequentially from the load lock chamber through a gate valve into an adjacent ultrahigh vacuum (base pressure $4 \times 10^{-10} \mathrm{Torr}$ ) analysis chamber.

The optical source for UPS was the He I $(21.2 \mathrm{eV})$ line from a differentially pumped VSW UV-10 discharge lamp. The helium discharge pressure was employed to discriminate against the $\mathrm{He}$ II $(40.8 \mathrm{eV})$ line present in the helium discharge. During the UPS measurements, the analysis chamber pressure was $2-4 \times 10^{-9}$ Torr. The angle integrated kinetic energy distribution of the photoemitted electrons was measured with a PHI 15-255 GAR double pass cylindrical mirror analyzer operated in the retarded mode with an instrumental resolution of $\pm 0.175 \mathrm{eV}$. The kinetic energy distribution of the electrons provides a surface sensitive (4-5 $\AA$ ) measurement of the joint density of states of the valence band. The samples were outgassed in situ at $160{ }^{\circ} \mathrm{C}$ using radiative heating from a resistive filament mounted behind the wafer block. UPS spectra were obtained at ground potential and with a perpendicularly applied electric field. The front surface of the sample is aligned parallel to the front surface of the analyzer which is at ground potential. The electrically isolated sample wafer block is shorted to ground for ground potential analysis and negatively biased with an external voltage supply for applied field analysis. The electric field is directed across the vacuum gap between the sample and the analyzer and is perpendicular to the sample surface. The photoemitted electrons are shifted in energy by the applied voltage.

The structural and electronic properties were investigated using first-principles density functional calculations. Our first- principles calculations are based on dispersion corrected density functional theory with general gradient approximation (GGA) for exchange-correlation potential. ${ }^{35} \mathrm{We}$ employed the dispersion correction with the GGA using the Tkatchenko-Scheffler (TS) scheme, which exploits the relationship between polarizability and volume. ${ }^{36}$ The TS dispersion correction accounts for the relative variation in dispersion coefficients of differently bonded atoms by weighting values taken from the high-quality first-principles database with atomic volumes derived from partitioning of the self-consistent electronic density ${ }^{36}$ Although the local density approximation (LDA) approach provides qualitatively correct pictures and remains the popular choice for investigations of electric-field effects ${ }^{26-29}$ our calculations reveal that the dispersion corrected GGA with the exchange correlation of Perdew-Burke-Ernzerhof (PBE), ${ }^{35,37}$ approximates the interlayer distance in graphite better than the GGA PBE itself and the local density approximation (LDA) approach.

We have calculated the layer distance of graphite with different exchange correlation functionals. While the LDA method gives short distances compared to the experimental value, the layer distance extracted from dispersion-corrected GGA is closer to the experimental value. Calculations using various LDA exchange-correlation functionals yield a value of $3.10 \AA$ for the layer distance. The underestimate of the LDA layer distance remains unsettled with the inclusion of dispersion correction in the LDA approach. On the other hand, GGA PBE has a very weak bonding. By contrast, the dispersion-corrected PBE predicts a layer distance of $3.29 \AA$, in good agreement with the experimental value of $3.35 \AA$.

A supercell with a vacuum space of $16 \AA$ normal to the graphene plane was used. The effect of the electric field was studied by adding a potential via the dipolar nuclear charges. ${ }^{37}$ For the graphite structure, we found that the slab system with more than 8 layers leads to the convergence of the electronic DOS and band structure. A supercell with a vacuum space of $10 \AA$ normal to the layers was employed. A kinetic energy change of $3 \times 10^{-4} \mathrm{eV}$ in the orbital basis and appropriate Monkhorst-Pack $k$-point grids of $6 \times 6 \times 1$ for twisted bilayer graphene and $16 \times 16 \times 1$ for graphite were sufficient to converge with the integration of the charge density. The optimization of the atomic positions proceeds until the change in energy is less than $1 \times 10^{-5} \mathrm{eV}$ per cell.

\section{Acknowledgements}

The authors are indebted to W. de Heer, C. Berger, and Y. Hu for providing the MEG sample for this study, to E. Conrad and M. Y. Chou for fruitful discussions, and to M. E. Walker for technical assistance in the UPS. This work was supported by the National Science Foundation under grants PREM DMR0934142 (MDW, DWH \& XQW) and MRSEC DMR-0820382 (DWH), and the Air Force Office of Scientific Research under grant FA9550-10-1-0254 (DKS and XQW).

\section{Notes and references}

1 A. K. Geim and K. S. Novoselov, Nat. Mater., 2007, 6, 183.

2 K. S. Novoselov, A. Geim, S. Morozov, D. Jiang, Y. Zhang, S. Dubonos, I. Grigorieva and A. Firsov, Science, 2004, 306, 666.

3 K. S. Novoselov, A. Geim, S. Morozov, D. Jiang, M. Katsnelson, I. Grigorieva, S. Dubonos and A. Firsov, Nature, 2005, 438, 197. 
4 R. R. Nair, P. Blake, A. N. Grigorenko, K. S. Novoselov, T. J. Booth, T. Stauber, N. M. R. Peres and A. K. Geim, Science, 2008, 320, 1308.

5 F. Schedin, A. K. Geim, S. V. Morozov, E. W. Hill, P. Blake, M. I. Katsnelson and K. S. Novoselov, Nat. Mater., 2007, 6, 652.

6 F. Schwierz, Nat. Nanotechnol., 2010, 5, 487.

7 A. A. Balandin, S. Ghosh, W. Bao, I. Calizo, D. Teweldebrhan, F. Miao and C. N. Lau, Nano Lett., 2008, 8, 902.

8 Q. Shao, G. Liu, D. Teweldebrhan and A. A. Balandin, Appl. Phys. Lett., 2008, 92, 202108.

9 A. K. Geim, S. V. Morozov, E. W. Hill, P. Blake, M. I. Katsnelson and K. S. Novoselov, Nat. Mater., 2007, 6, 652.

10 X. Wang, L. Zhi and K. Mullen, Nano Lett., 2008, 8, 323.

11 M. K. Singh, E. Titus, G. Gonçalves, P. A. A. P. Marques, I. Bdikin, A. L. Kholkin and J. J. A. Gracio, Nanoscale, 2010, 2, 700.

12 S. Gilje, S. Han, M. Wang, K. L. Wang and R. B. Kaner, Nano Lett., 2007, 7, 3394.

13 W. A. de Heer, C. Berger, M. Ruan, M. Sprinkle, X. Li, Y. Hu, B. Zhang, J. Hankinson and E. H. Conrad, Proc. Natl. Acad. Sci. U. S. A., 2011, 108, 16900.

14 M. M. Orlita, C. M. Faugeras, P. M. Plochocka, P. K. Neugebauer, G. M. Martinez, D. K. Maude, A. M. Barra, M. A. Sprinkle, C. M. Berger and W. A. de Heer, et al., Phys. Rev. Lett., 2009, 101, 267601.

15 K. V. Emtsev, A. Bostwick, K. Horn, J. Jobst, G. L. Kellogg, L. Ley, J. L. McChesney, T. Ohta, S. A. Reshanov and J. Röhrl, et al., Nat. Mater., 2009, 8, 203.

16 A. Reina, X. Jia, J. Ho, D. Nezich, H. Son, V. Bulovic, M. S. Dresselhaus and J. Kong, Nano Lett., 2009, 9, 30.

17 D. K. Samarakoon, Z. Chen, C. Nicolas and X.-Q. Wang, Small, 2011, 7, 965.

18 B. Partoens and F. M. Peeters, Phys. Rev. B: Condens. Matter Mater. Phys., 2006, 74, 075404.

19 S. Latil and L. Henrard, Phys. Rev. Lett., 2006, 97, 036803.

20 A. Grueneis, C. Attaccalite, L. Wirtz, H. Shiozawa, R. Saito, T. Pichler and A. Rubio, Phys. Rev. B: Condens. Matter Mater. Phys., 2008, 78, 205425.
21 A. A. Avetisyan, B. Partoens and F. M. Peeters, Phys. Rev. B: Condens. Matter Mater. Phys., 2010, 81, 115432.

22 Y. Zhang, T.-T. Tang, C. Girit, Z. Hao, M. C. Martin, A. Zettl, M. F. Crommie, Y. R. Shen and F. Wang, Nature, 2009, 459, 820.

23 M. F. Craciun, S. Russo, M. Yamamoto, J. B. Oostinga, A. F. Morpurgo and S. Thrucha, Nat. Nanotechnol., 2009, 4, 383.

24 K. F. Mak, M. Y. Sfeir, J. A. Misewich and T. F. Heinz, Proc. Natl. Acad. Sci. U. S. A., 2010, 107, 14999.

25 H. M. Wang, Y. H. Wu, Z. H. Ni and Z. X. Shen, Appl. Phys. Lett., 2008, 92, 053504.

26 B. Sahu, H. Min, A. H. MacDonald and S. K. Banerjee, Phys. Rev. B: Condens. Matter Mater. Phys., 2008, 78, 045404.

27 Z. Zhang, C. Chen, X. C. Zeng and W. Guo, Phys. Rev. B: Condens. Matter Mater. Phys., 2010, 81, 155428.

28 B. Sahu, H. Min and S. K. Banerjee, Phys. Rev. B: Condens. Matter Mater. Phys., 2010, 82, 115426.

29 D. K. Samarakoon and X.-Q. Wang, ACS Nano, 2010, 4, 4126.

30 Z. Chen and X.-Q. Wang, Phys. Rev. B: Condens. Matter Mater. Phys., 2011, 83, 081405.

31 E. J. Mele, Phys. Rev. B: Condens. Matter Mater. Phys., 2010, 81, 161405.

32 M. Kindermann and E. J. Mele, Phys. Rev. B: Condens. Matter Mater. Phys., 2011, 84, 161406(R).

33 B. Partoens and F. M. Peeters, Phys. Rev. B: Condens. Matter Mater. Phys., 2007, 75, 193402.

34 D. Sun, C. Divin, J. Rioux, J. E. Sipe, C. Berger, W. A. de Heer, P. N. First and T. B. Norris, Nano Lett., 2010, 10, 1293.

35 J. Perdew, K. Burke and M. Ernzerhof, Phys. Rev. Lett., 1996, 77, 3865 .

36 A. Tkatchenko and M. Scheffler, Phys. Rev. Lett., 2009, 102, 073005.

37 DMol3, Accelrys Software Inc., San Diego, CA, 2011.

38 J. N. Coleman, et al., Science, 2011, 331, 568.

39 Y. Ma, Y. Dai, M. Guo, C. Niu and B. Huang, Nanoscale, 2011, 3, 3883.

40 Y. Ma, Y. Dai, W. Wei, C. Niu, L. Yu and B. Huang, J. Phys. Chem. C, 2011, 115, 20237. 